\title{
Performance Analysis of a Hybrid DS/SFH CDMA System Using Analytical and Measured Pico Cellular Channels
}

\author{
Fevzi Çakmak, Student Member, IEEE, René G. A. Rooimans, Member, IEEE, \\ and Ramjee Prasad, Senior Member, IEEE
}

\begin{abstract}
This paper presents the throughput and delay analysis of a packet-switched code division multiple access (CDMA) network based on the hybrid direct sequence (DS)/slow frequency hopping (SFH) spread-spectrum multiple access (SS MA) technique with $Q-, B$-, and $D$-PSK modulation using analytical and measured pico cellular channels. The performance of the hybrid DS/SFH, DS, and SFH multiple access techniques have been compared in a pico cellular personal communications network (PCN) environment. Multipath and multiple access interference are considered. The performance is evaluated for a given delay spread and a fixed bandwidth. The effects of forward error correction (FEC) coding and diversity techniques, such as selection diversity and maximal ratio combining on the performance, are also investigated.
\end{abstract}

\section{INTRODUCTION}

C ODE division multiple access (CDMA) is a strong future candidate as a multiple access scheme for a pico cellular personal communications network (PCN). CDMA is not only attractive from the point of multiple access capabilities, which is needed when a large number of users want to transmit simultaneously, but also for combating multipath fading, which occurs in pico cellular radio channels. In the family of CDMA, direct sequence (DS) and slow frequency hopping (SFH) techniques have their individual features. The DS CDMA technique is a good answer to the problem of multipath fading [1]-[6]; it suffers, however, from the near-far effect. On the other hand, the SFH CDMA technique is more sensitive to multipath interference, but suffers less from the near-far effect. As a consequence the combination of direct sequence and slow frequency hopping, hybrid DS/SFH CDMA, is an attractive solution for combating the multipath effect, and the multiple access interference, and for reducing the near-far problem [7]-[9].

The present paper has the following three main technical contributions. First, the paper evaluates the throughput and the delay characteristics of a packet-switched CDMA network based on hybrid DS/SFH, DS, and SFH. Secondly, the performance of of hybrid DS/SFH CDMA is evaluated and compared using $Q-, B$-, and $D$-PSK modulation. These two evaluations are performed using an analytical model in which

Manuscript revised December 8, 1995. This paper was presented in parts at PIMRC'93, PIRMC'94, GLOBECOM'94, and GLOBECOM'95.

The authors are with the Telecommunications and Traffic Control Systems Group, Delft University of Technology, 2600 GA Delft, The Netherlands.

Publisher Item Identifier S 0733-8716(96)02520-6. the radio channel has been modeled as a slow Rician-fading channel. Thirdly, the throughput and delay of hybrid DS/SFH CDMA are obtained using the measured delay profiles in a pico cellular environment and the performance is compared with the results obtained with the analytical model.

An analytical framework of random access spread-spectrum networks has been presented in [10], in which link-level and topological issues are discussed. We use this analytical framework in this paper and we additionally consider the physical level of communication in the CDMA network. This means that the effects of fading, the use of various modulation types such as $Q-, B$-, and $D$-PSK, the use of forward error correction (FEC) codes and the effect of diversity on the throughput and delay performance of the CDMA network are evaluated. The results of this paper can be applied to develop pico cellular wireless personal communications systems and wireless local area networks (LAN's).

The paper is structured as follows. In Section II, analytical and measured channel models are described. In Section III, the expression for the packet success probabilities for the different modulation types are given. In Section IV, the performance results are given and discussed. Finally conclusions are given in Section $\mathrm{V}$.

\section{ChanNel MODELS}

We consider a centralized network with $C$ users and one coordinating base station, consisting of $C$ spread-spectrum transceivers (transmitters/receivers). Each transmitter/receiver pair is identified by its own spread-spectrum code and hopping pattern. So, from a topological viewpoint we are dealing with paired-off users, which means there is no topological "competition" between active transmitters. The multi-user interference is the only disruptive interaction between pairs [10]. The network has the following characteristics: i) Data is transmitted in packets of $N_{p}$ bits; ii) the system is slotted; iii) a time slot is equal to the packet duration; iv) the system has a positive acknowledgment scheme. In order to be able to give some closed expressions for the throughput, we have to make the following five assumptions: i) All users are identical from a statistical point of view and the same holds for the transceivers at the base station; ii) the averaged received power at the base station is equal for each user; iii) the acknowledgment are almost costless from a capacity point of view, fully reliable and 
instantaneous; iv) the channel is memoryless; v) the system is in a stable state.

The link between the $k$ th user and the base station is characterized by its lowpass equivalent impulse response, which is given as

$$
h_{k}(t)=\sum_{l=1}^{L} \beta_{k l} \delta\left(t-\tau_{k l}\right) \exp \left(j \gamma_{k l}\right)
$$

where $k l$ refers to the $l$ th path of user $k$.

\section{A. Analytical Channel Model Assuming Identically Distributed Path Gains}

In case of the analytical independent identically distributed (i.i.d.) channel the following assumptions are made concerning the channel parameters: i) the path gains $\beta_{k l}$ are Rician distributed; ii) the delays $\tau_{k l}$ are uniformly distributed random variables over $[0, T]$; iii) the phases $\gamma_{k l}$ are uniformly distributed over $[0,2 \pi]$. The channel is assumed to be of the slow fading type, which means that the random variables do not change considerably for the duration of one packet.

The probability density function (pdf) of the Rician distributed path gains $\beta_{k l}$ is given by

$$
p_{\beta}(r)=\frac{r}{\sigma_{r}^{2}} \exp \left(-\frac{r^{2}+A^{2}}{2 \sigma_{r}^{2}}\right) I_{0}\left(\frac{r A}{\sigma_{r}^{2}}\right)
$$

with $r \geq 0$ and $A \geq 0$. Here $I_{0}()$ represents the modified Bessel function of the first kind and zero order. The Rice parameter $R$ is defined as the ratio of the power associated with the specular signal component $A^{2} / 2$ and the power associated with the scattered components $\sigma_{r}^{2}$. This parameter incorporates the radio characteristics of the environment.

When the signal bandwidth is much larger than the coherence bandwidth of the channel, then the channel is said to be frequency selective. This means that different paths can be resolved by the receiver. The maximum number of resolvable paths $L$ is given by

$$
L=\left\lfloor\frac{T_{m}}{T_{c}}\right\rfloor+1=\left\lfloor N R_{b} T_{m}\right\rfloor+1
$$

where $T_{m}$ denotes the rms delay spread, $T_{c}$ the chip time, $N$ the spreading code length ( $N_{q}$ for $Q$-PSK, $N_{b}$ for $B$-PSK, and $N_{d}$ for $D$-PSK), and $R_{b}$ the channel bit rate.

\section{B. Channel Model Using the Pico Cellular Measured Power Delay Profiles}

The power delay profile measurements have been carried out in the frequency bands $2.4,4.75$, and $11.5 \mathrm{GHz}$ in an indoor environment [11]. In the analytical channel model we have described the path gains by a Rician pdf, while in the measured pico cellular channel model we have used the true measured values of the path gains for the calculations. This is a more practical situation than assuming the path gains to be Rician distributed. An measured power delay (MPD) profile is shown in Fig. 1. The peaks of the profile shown in Fig. 1 are estimated. The estimation is based on the fact that each local maximum in the original profile represents a ray. Due to the inherent diversity of the direct sequence spread

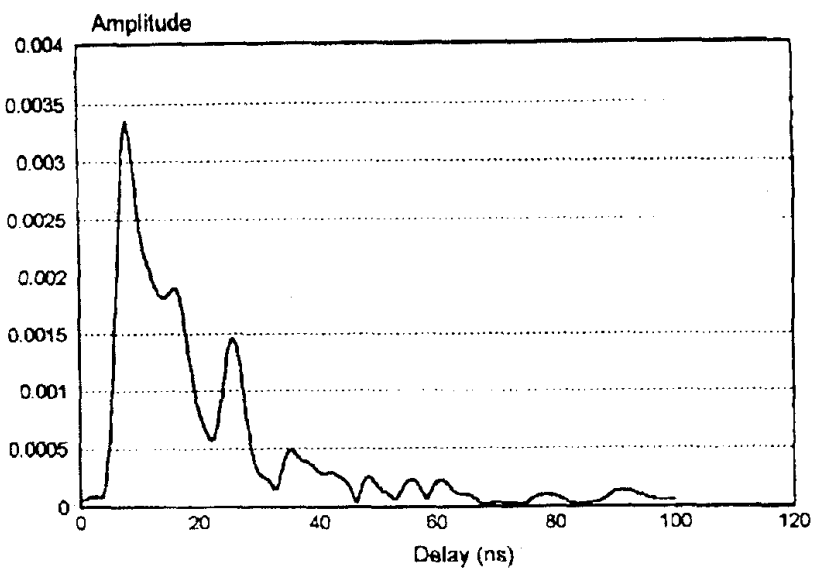

Fig. 1. Measured MDP.

spectrum (DS/SS), the delayed versions of the initial pulse can be grouped in clusters which can be resolved independently. Two signals having a time difference less than the chip time $T_{c}$ are treated as a single signal. These two signals can be combined by means of a vectorial summation. For example, if we assume that the delay, amplitude and phase of ray 1 are respectively denoted as $\tau_{1}, \beta_{1}$, and $\phi_{1}$, and the delay, amplitude, and phase of ray 2 are respectively denoted as $\tau_{2}, \beta_{2}$, and $\phi_{2}$, then the delay, amplitude, and phase of the resulting signal are given by

$$
\begin{aligned}
\tau_{r} & =\frac{\beta_{1} \tau_{1}+\beta_{2} \tau_{2}}{\beta_{1}+\beta_{2}} \\
\beta_{r} & =\sqrt{\left(\beta_{1} \cos \phi_{1}+\beta_{2} \cos \phi_{2}\right)^{2}+\left(\beta_{1} \sin \phi_{1}+\beta_{2} \sin \phi_{2}\right)^{2}}
\end{aligned}
$$

and

$$
\phi_{r}=\arctan \left(\frac{\beta_{1} \sin \phi_{1}+\beta_{2} \sin \phi_{2}}{\beta_{1} \cos \phi_{1}+\beta_{2} \cos \phi_{2}}\right) .
$$

If we assume selection diversity, we lock on the strongest received path. If no diversity is being used, we lock on the first received path.

\section{PaCKet Success Probability}

The packet success probability from a particular transmitter's viewpoint given $(k-1)$ other packets in the channel is represented by $P_{k}(k)$. We can interpret the packet success probability as the probability that the packet is received without any bit errors. This is the probability which incorporates the physical aspects, such as modulation type and the channel statistics. Although the bit errors in a packet are often highly correlated, we assume independent bit errors in a packet for the sake of mathematical convenience. When channel coding is used, this is a reasonable assumption, because in that case the information bits are interleaved by coding bits which creates less correlation among the information bits. For independent bit errors in a packet we can write [4]

$$
P_{k}(k)=\left[1-P_{e}(k)\right]^{N_{p}}
$$

where $P_{e}(k)$ denotes the average bit error probability as a function of the number simultaneously transmitting users $k$. 
The expression for $P_{e}(k)$ is determined by the modulation type.

In order to determine the packet success probability, we first have to consider the average bit error probability, according to (7). We have to keep in mind that we consider the channel to be of the slow fading type, which means that the channel statistics do not change considerably for the duration of one packet. First the packet success probability is derived for $D$-PSK, $Q$-PSK, and $B$-PSK modulation using the analytical model and then it is derived for $B$-PSK modulation using the measured delay profile.

\section{A. Packet Success Probability and Bit Error \\ Probability in Case of D-PSK}

In case of hybrid DS/SFH with D-PSK modulation and selection diversity the conditional bit error probability as a function of the number of simultaneously transmitting users is given by

$$
P_{e}\left(k \mid \beta_{\max }, \tau_{k l}, L\right)=\sum_{n_{i}=0}^{k-1} P_{e}\left(n_{i} \mid \beta_{\max }, \tau_{k l}, L\right) P\left(n_{i}\right) .
$$

Here $P\left(n_{i}\right)$ represents the probability of having $n_{i}$ active interferers out of $k-1$ active transmitters and is given by

$$
P\left(n_{i}\right)=\left(\begin{array}{c}
k-1 \\
n_{i}
\end{array}\right)\left(\frac{1}{q}\right)^{n_{i}}\left(1-\frac{1}{q}\right)^{k-1-n_{i}}
$$

where $q$ is the number of frequencies in the hopping pattern. In case of selection diversity and additive white Gaussian noise (AWGN) the conditional bit error probability $P_{e}\left(n_{i} \mid\right.$ $\left.\beta_{m}, \tau_{k l}, L\right)$ is given by [6]

$$
\begin{aligned}
P_{e}\left(n_{i} \mid \beta_{m},\left\{\tau_{k l}\right\}, L\right)= & Q(a, b)-\frac{1}{2}\left(1+\frac{\mu}{\sqrt{\mu_{0} \mu}-1}\right) \\
& \times \exp \left(-\frac{a^{2}+b^{2}}{2}\right) I_{0}(a b)
\end{aligned}
$$

where $Q()$ denotes the Marcum $Q$-function. Here we have assumed fixed delays, phase angles bits, and considered that the maximum of the envelope has been found (all other signals are seen as noise). According to [6] the $\mu$-parameters in (10), representing the variances and the covariance, respectively, of the matched filter output of the receiver, are assumed to be random Gaussian variables, which are characterized by a Gaussian distribution. The expressions for the $\mu$-parameters are given here below

$$
\begin{aligned}
\mu_{0}= & \frac{P}{8} \sum_{k=1}^{n_{i}}\left[E\left(X_{k}^{2}\right)+E\left(\hat{X}_{k}^{2}\right)+E\left(Y_{k}^{2}\right)+E\left(\hat{Y}_{k}^{2}\right)\right] \\
& +\frac{P}{4}\left[E\left(X_{1} \hat{X}_{1}\right)+E\left(Y_{1} \hat{Y}_{1}\right)\right]+2 \sigma_{N}^{2} \\
\mu_{-1}= & \frac{P}{8} \sum_{k=1}^{n_{i}}\left[E\left(X_{k}^{2}\right)+E\left(\hat{X}_{k}^{2}\right)+E\left(Y_{k}^{2}\right)+E\left(\hat{Y}_{k}^{2}\right)\right] \\
& +2 \sigma_{N}^{2} \\
\mu= & \frac{P}{8} \sum_{k=1}^{n_{i}}\left[E\left(X_{k} \hat{X}_{k}\right)+E\left(Y_{k} \hat{Y}_{k}\right)\right] \\
& +\frac{P}{8}\left[E\left(\hat{X}_{k}\right)+E\left(\hat{Y}_{k}\right)\right]
\end{aligned}
$$

where $X_{1}, X_{k}, \hat{X}_{1}$, and $\hat{X}_{k}$ are given by

$$
\begin{aligned}
& X_{1}=\sum_{l, l \neq j} \beta_{1 l} \cos \left(\psi_{1 l}\right) R_{1 l}\left(\tau_{1 l}\right) \\
& X_{k}=\sum_{l} \beta_{k l} \cos \left(\psi_{k l}\right) R_{k l}\left(\tau_{k l}\right) \\
& \hat{X}_{1}=\sum_{l, l \neq j} \beta_{1 l} \cos \left(\psi_{1 l}\right) \hat{R}_{1 l}\left(\tau_{1 l}\right) \\
& \hat{X}_{k}=\sum_{l} \beta_{k l} \cos \left(\psi_{k l}\right) \hat{R}_{k l}\left(\tau_{k l}\right)
\end{aligned}
$$

respectively (similar expressions for $Y$ ). The partial correlation functions $R_{1 k}$ and $\hat{R}_{1 k}$ as well as $a$ and $b$ are defined in [5]. Substitution of the conditional bit error probability (10) in (7) yields the conditional packet success probability. The conditioning on the random variables is now removed by weighting the conditional success probability with the probability density functions of the $\mu$-parameters and with the Rician fading statistics, i.e., the probability density function of the maximal path gain $\beta_{m}$. For an order of diversity equal to $M$ and assuming that all path gains are equally distributed independent Rician variables, this pdf becomes

$$
\begin{aligned}
f_{\beta_{m}}\left(\beta_{m}\right) & \\
= & M\left[\int_{0}^{\beta_{m}} \frac{z}{\sigma_{r}^{2}} \exp \left(-\frac{A^{2}+z^{2}}{2 \sigma_{r}^{2}}\right) \cdot I_{0}\left(\frac{A_{z}}{\sigma_{r}^{2}}\right) d z\right]^{(M-1)} \\
& \cdot \frac{\beta_{m}}{\sigma_{r}^{2}} \exp \left(-\frac{A^{2}+\beta_{m}^{2}}{2 \sigma_{r}^{2}}\right) \cdot I_{0}\left(\frac{A \beta_{m}}{\sigma_{r}^{2}}\right) .
\end{aligned}
$$

The unconditional packet success probability is then given by

$$
\begin{gathered}
P_{k}^{\prime}(k)=\int_{\mu} \int_{\mu_{-1}} \int_{\mu} \int_{\beta_{m}}\left[1-P_{e}\left(k \mid \beta_{m}, \mu_{0}, \mu_{-1}, \mu\right)\right]^{N_{p}} \\
\cdot f_{\beta_{m}}\left(\beta_{m}\right) f_{\mu_{0}}\left(\mu_{0}\right) f_{\mu_{-1}}\left(\mu_{-1}\right) \\
\cdot f_{\mu}(\mu) d \beta_{m} d \mu d_{\mu_{-1}} d_{\mu_{0}} .
\end{gathered}
$$

\section{B. Packet Success Probability and Bit Error} Rate in Case of $B$ - and Q-PSK

In case of $B$ - and $Q$-PSK modulation, the conditional bit error probability is given by

$$
P_{e}(k \mid \alpha)=\sum_{n_{i}=0}^{k-1} P_{e}^{B, Q}\left(n_{i} \mid \alpha\right) P\left(n_{i}\right)
$$

where $P_{e}^{B}$ and $P_{e}^{Q}$ are the bit error probabilities for $B$-PSK and Q-PSK modulation, respectively. We have derived these bit error probabilities for both selection diversity and maximal ratio combining.

1) Selection Diversity: Selection diversity means that the largest of a group of $M$ signals carrying the same information is selected. The order of diversity $M$ equals the number of antennas times the number of paths. The bit error probabilities in case of selection diversity are given as

$$
\begin{aligned}
P_{e}^{B}\left(n_{i} \mid \alpha_{\max }\right)= & \frac{1}{2} \operatorname{erfc}\left\{\left[\left(\frac{E_{b}\left(2 \sigma_{r}^{2}+A^{2}\right)}{N_{0}}\right)^{-1} \frac{1}{\alpha_{1 j}^{2}}\right.\right. \\
& \left.\left.+\frac{2 L}{3 N \alpha_{1 j}^{2}}\left(1+n_{i}\right)\right]^{-0,5}\right\}
\end{aligned}
$$


and

$$
\begin{aligned}
P_{e}^{Q}\left(n_{i} \mid \alpha_{\max }\right)= & \frac{1}{2} \operatorname{erfc}\left\{\left[\left(\frac{E_{b}\left(2 \sigma_{r}^{2}+A^{2}\right.}{N_{0}}\right)^{-1} \frac{1}{\alpha_{1 j}^{2}}\right.\right. \\
& \left.\left.+\frac{L}{3 N \alpha_{1 j}^{2}}\left(1+n_{i}\right)\right]^{-0,5}\right\}
\end{aligned}
$$

for $B$-PSK and $Q$-PSK, respectively. Substitution of the conditional bit error probabilities given in (16) or (17) in (7) yields the conditional packet success probability for $B$ or $Q$-PSK modulation. Similar to $D$-PSK, the conditioning is removed by weighting the conditional bit error probability, given in (15), with the pdf of the normalized maximal path gain $\alpha_{m}$. This pdf is obtained by a change of variables in (11) in the following way: $\alpha=\beta / \sqrt{A^{2}+2 \sigma_{r}^{2}}$ and $y=z / \sqrt{A^{2}+2 \sigma_{r}^{2}}$. For the pdf of the normalized maximum path gain we then find

$$
\begin{aligned}
& f_{\alpha_{\max }}(\alpha)=M\left[\int_{0}^{\alpha} 2 y(1+R) \exp \left(-R-(1+R) y^{2}\right)\right. \\
&\left.\times I_{0}(2 \sqrt{R(1+R) y}) d y\right]^{(M-1)} \\
& \cdot 2 \alpha(1+R) \exp \left(-\alpha^{2}(1+R)-R\right) \\
& \times I_{0}(2 \sqrt{R(1+R) \alpha}) .
\end{aligned}
$$

The unconditional packet success probability is now given by

$$
P_{k}^{\prime}(k)=\int_{\alpha_{m}}\left[1-P_{e}\left(k \mid \alpha_{m}\right)\right]^{N_{p}} f_{\alpha_{m}}\left(\alpha_{m}\right) d \alpha_{m} .
$$

2) Maximal Ratio Combining: In case of maximal ratio combining, the contributions of the several resoluted paths are added together. There is a compensation for the phase shift and a weighting associated with the signal strength. The bit error probability for $B$-PSK and Q-PSK modulation are given, respectively, as

$$
\begin{aligned}
P_{e}^{B}\left(n_{i} \mid \alpha_{\max }\right)=\frac{1}{2} \operatorname{erfc}\{ & {\left[\left(\frac{E_{b}\left(2 \sigma_{r}^{2}+A^{2}\right)}{N_{0}}\right)^{-1} \frac{1}{M v}\right.} \\
& \left.\left.+\frac{2 L}{3 N M v}\left(1+n_{i}\right)\right]^{-0,5}\right\}
\end{aligned}
$$

and

$$
\begin{aligned}
P_{e}^{Q}\left(n_{i} \mid \alpha_{\max }\right)=\frac{1}{2} \operatorname{erfc}\{ & {\left[\left(\frac{E_{b}\left(2 \sigma_{r}^{2}+A^{2}\right)}{N_{0}}\right)^{-1} \frac{1}{M v}\right.} \\
& \left.\left.+\frac{L}{3 N M v}\left(1+n_{i}\right)\right]^{-0,5}\right\}
\end{aligned}
$$

where $v$ is a normalized variable and is defined as $v=$ $t_{n} / M\left(2 \sigma_{r}^{2}+A^{2}\right)$ with $t_{n}=\sum_{m=1}^{M} \beta_{1 m}^{2}$. In order to remove the channel statistics we have to average these bit error probabilities with the pdf of $v$, which is given by

$$
\begin{aligned}
f(v)= & M[1+R]\left[v\left(1+\frac{1}{R}\right)\right]^{M-1 / 2} \\
& \times \exp (-M R-M v(1+R)) \\
& \cdot I_{M-1}(2 M \sqrt{v R(1+R)}) .
\end{aligned}
$$

The unconditional packet success probability is now given by

$$
P_{k}^{\prime}(k)=\int_{v}[1-P e(k \mid v)]^{N_{p}} f_{v}(v) d v .
$$

\section{Packet Success Probability and Bit Error Rate in Case of the Measured Power Delay Profile for B-PSK Modulation}

In case of the measured pico cellular channel model we do not approximate the path gains, delays and phase shifts by random variables. In fact we use the true measured values for the calculations. The average bit error probability for the measured pico cellular channel model is given as (24) (shown at the bottom of the page). If we look at the downlink, the channel is the same for all $k$ users. Then the subscript $k$ in (24) disappears. The packet success probability can now be calculated by using (7).

\section{Performance Results}

The performance has been investigated in terms of throughput and delay. We have used the definition for the throughput $S$, [4], [10], as the expected number of successfully received packets per time slot, given by

$$
S=\sum_{k=1}^{C} k p_{k} P_{k}^{\prime}(k)
$$

where $p_{k}$ is the probability density function of the composite arrivals in a given time slot. For identical, independent, and a finite number of users, this pdf is a binomial distribution given by

$$
p_{k}=\left(\begin{array}{l}
C \\
k
\end{array}\right)\left[\frac{G}{C}\right]^{k}\left[1-\frac{G}{C}\right]^{C-k}
$$

where the offered traffic $G$ is defined as the average number of transmissions (new plus retransmitted packets) per time slot by $C$ users. The average normalized delay $D$ of the system is defined as the average number of time slots between the generation and the successful reception of a packet. According to [4] the normalized delay is given by

$$
D=1.5+\left[\frac{G}{S}-1\right](\lfloor\delta+1\rfloor+1)
$$

where $(G / S-1)$ represents the average number of retransmissions needed for a packet to be received successfully and $\delta$ denotes the mean of the retransmission delay. The round trip propagation delay can be neglected in a pico cellular environment.

$$
P_{e}(k)=\sum_{n_{i}=0}^{k-1} \frac{1}{2} \operatorname{erfc}\left\{\sqrt{\frac{\frac{P T^{2}}{8} \beta_{1 j}^{2}}{\frac{P T^{2}}{6 N} \sum_{l, l \neq j} \beta_{1 l}^{2} \cos ^{2}\left(\Psi_{1 l}\right)+n_{i} \frac{P T^{2}}{6 N} \sum_{l=1}^{L} \beta_{k l}^{2} \cos ^{2}\left(\Psi_{k l}\right)+\frac{N_{0} T}{8}}}\right\} \cdot P\left(n_{i}\right) .
$$


The throughput $S$ and delay $D$ are computed using (25)-(27) and the analytical expressions of Section III for hybrid DS/SFH CDMA. The throughput and delay are also evaluated for DS and SFH CDMA by adapting the hybrid DS/SFH analytical expressions. In the case of direct sequence, all users use the same carrier frequency; hence, the number of hopping frequencies $q$ then becomes equal to one. The number of active interferers $n_{i}$ is then equal to the number of active transmitters $k$. For the probability of having $n_{i}$ active interferers we then have

$$
P\left(n_{i}\right)=1 \text {. }
$$

In case of SFH, the direct sequence part has to be left out of the model by taking $N=1$. The number of resolvable paths, $L$ in this case, is equal to one, because there is no inherent spread-spectrum anymore.

We have considered two types of FEC codes, viz. the $(7,4)$ Hamming code and the $(23,12)$ Golay code. The figures between brackets $(n, k)$ means that $k$ source bits are transformed into a block of $n$ channel bits by coding. The conditional bit error probability at the hard decision output is given by

$$
P_{\text {ecl }}=\frac{1}{n} \sum_{m=t+1}^{n} m\left(\begin{array}{c}
n \\
m
\end{array}\right) P_{e}^{m}\left(1-P_{e}\right)^{n-m}
$$

where $t$ denotes the number of errors the code can correct and $P_{e}$ the conditional bit error probability at the hard decision output.

\section{A. Analytical Channel Model Assuming Identically Distributed Path Gains}

For the comparison of the three modulation types $D$-PSK, $Q$-PSK, and $B$-PSK we have kept the bandwidth, information bit rate and the rms delay spread fixed. This means that the ratio $N q / T$ has to be the same for the three systems. Unless stated otherwise we have chosen the parameters such that the ratio $N q / T$, which is proportional to the transmission bandwidth, equals approximately $200 \mathrm{MHz}$. This is a practical value for spread-spectrum communications systems.

Since the bit rates for $B$-PSK and $D$-PSK are equal to $1 / T$ and for $Q$-PSK equal to $1 / T_{q}=1 / 2 T$, the bit rate constraint leads to $T_{q}=2 T$, where $T_{q}$ is the bit duration in case of $Q$-PSK and where $T$ is the bit duration in case of $B$ - and $D$-PSK. Assuming the same number of hopping frequencies, $q$ for the three systems, we finally have $N_{q}=2 N_{b}=2 N_{d}$, where $N_{q}, N_{b}$, and $N_{d}$ denote the spreading code lengths in case of $Q$-PSK, $B$-PSK, and $D$-PSK, respectively.

When FEC coding is used, we would like to keep the information bit rate $R_{i}$ the same. For the channel bit rate $R_{b}$ we can write $R_{b}=(n / k) R_{i}$. Since the ratio $n / k$ approximately equals two for the $(7,4)$ Hamming and $(23,12)$ Golay code, the channel bit rate should be doubled in case of FEC coding in order to have the information bit rate fixed. In all cases $R_{i}$ is taken $64 \mathrm{~kb} / \mathrm{s}$. We have considered a signal-to-noise (SNR) atio of $20 \mathrm{~dB}$ (in case of FEC coding this becomes $17 \mathrm{~dB}$ ). The packet length $N_{p}$ is taken equal to $1024 \mathrm{~b}$. In the analytical model we have assumed the rms delay spread $T_{m}$ to be equal to

\section{S [packets/slot]}

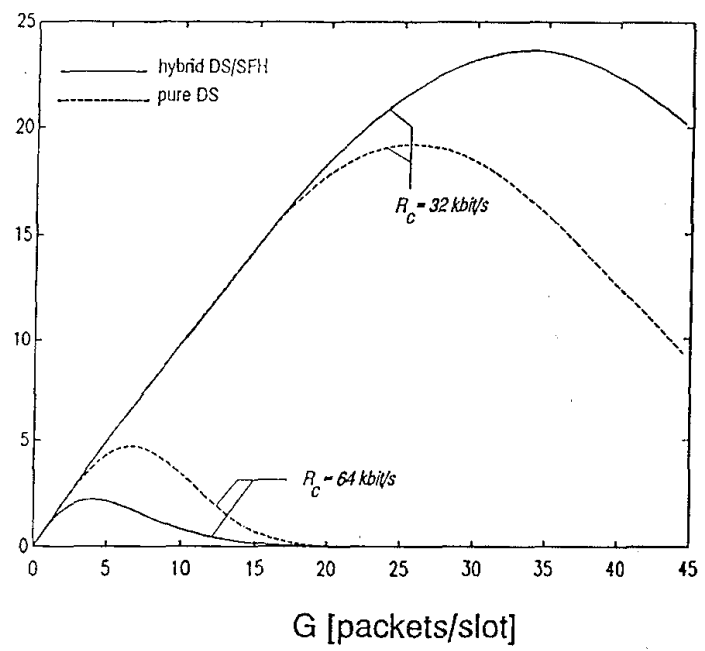

Fig. 2. Comparison of the throughput of hybrid DS/SFH CDMA and DS CDMA with $D$-PSK, with the bit rate as a parameter, using the analytical channel model. $R=6,8 \mathrm{~dB} ; M=4 ; q=2 ; N_{\mathrm{DS} / \mathrm{S}_{\mathrm{SFH}}}=127$; $N_{\mathrm{DS}}=2.55$

250 ns. The Rice parameter $R$, which characterizes the indoor environment is taken equal to $6.8 \mathrm{~dB}$.

In Table I we have presented the throughput $S$ normalized on the offered traffic $G$ for $Q-, B$-, and $D$-PSK modulation with the order of selection diversity $M$ as a parameter. It is seen that Q-PSK yields relatively the largest throughput and $D$-PSK relatively the poorest. It is obvious that selection diversity enhances the performance in comparison with nondiversity $(M=1)$.

Figure 2 depicts the throughput comparison for hybrid DS/SFH CDMA and DS CDMA for $D$-PSK modulation at fixed bandwidth of approximately $16 \mathrm{MHz}$ for two bit rates, viz. $32 \mathrm{~kb} / \mathrm{s}$ and $64 \mathrm{~kb} / \mathrm{s}$. It is seen that for a relatively low bit rate, which corresponds with a relatively small number of resolvable paths according to (3), the hybrid system yields a larger throughput than the DS system. For a higher bit rate and consequently a larger number of resolvable paths, pure DS performs better than hybrid DS/SFH with this parameter setting and the chosen bandwidth. The number of hopping frequencies should be increased in order to fully explore the capabilities of hybrid DS/SFH. In this case, where the number of hopping frequencies only equals two, DS outperformes hybrid DS/SFH. We also see that an increase in bit rate decreases the performance for both hybrid DS/SFH and pure DS.

Figure 3 shows the effect of FEC coding on the delay for hybrid DS/SFH with $Q$ - and $B$-PSK modulation at fixed bandwidth for the same number of hopping frequencies, but with varying $N . Q$-PSK modulation and $(23,12)$ Golay coding yields the best performance. In this case, the $(7,4)$ Hamming code does not improve the performance for both modulation techniques. Since the bandwidth is kept fixed in the noncoding and coding situation, the spreading code length $N$ is reduced by a half in case of coding. This means that the multi-user and multipath interference terms have a larger influence in 
$D$ [slots]

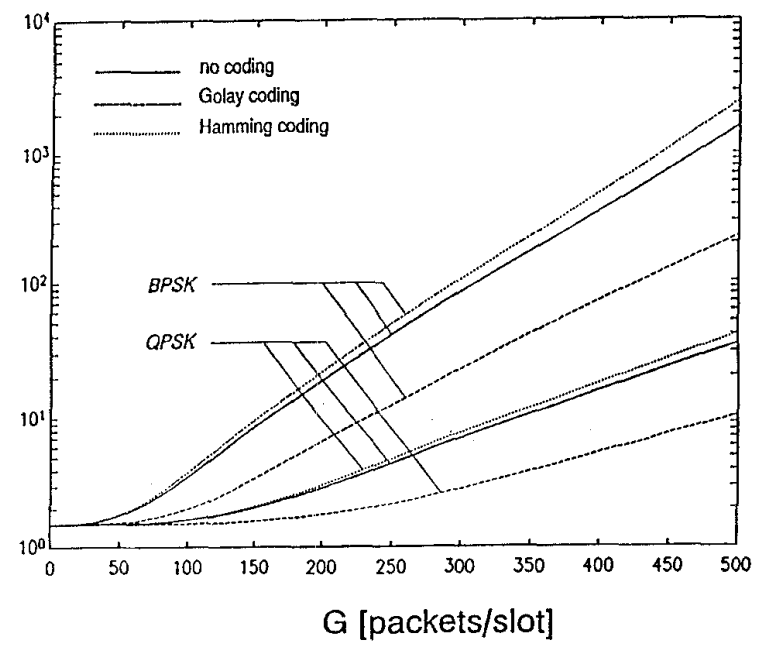

Fig. 3. Effect of FEC coding on the delay of hybrid DS/SFH CDMA with $B$ - and $Q$-PSK, using the analytical channel model. $M=4 ; q=10 ; L=5$; $N_{q}=255$ (coding); $N_{q}=511$ (no coding); $N_{b}=127$ (coding); $N_{b}=255$ (no coding).

that case [see (16) and (17)], which has to be overcome by the FEC codes. The Hamming code has a poorer coding gain than the Golay code and so the Hamming code isn't able to improve the performance in this case.

Figure 4 show the delay performance of hybrid DS/SFH CDMA with $B$-PSK modulation, selection diversity, maximal ratio combining, and order of diversity $M$ as a parameter. It is seen that maximal ratio combining is superior over selection diversity, which becomes more obvious for larger values of $M$. Maximal ratio combining uses all available paths to construct a decision variable, while selection diversity only uses the strongest path to come to a decision variable. In the case of maximal ratio combining, a more reliable decision can be made than in the case of selection diversity.

Figure 5 depicts the throughput comparison of hybrid DS/SFH CDMA with the throughput of the pure DS CDMA, for $B$-PSK and $Q$-PSK modulation without FEC coding. We can see that hybrid DS/SFH CDMA has a higher throughput than the pure DS CDMA. In this situation the number of hopping frequencies is large enough to outperform DS as opposed to Fig. 2. Although the multi-user and multipath interference terms are slightly larger with hybrid DS/SFH than in case of DS [according to (16) and (17)], the number of hopping frequencies is large enough to overcome the effect of the interference.

In Fig. 6 the throughput using hybrid DS/SFH is compared with the throughput using pure DS and SFH for $B$ - and $Q$-PSK modulation, with FEC coding. For the pure SFH technique the number of resolvable paths $L$ is equal to one. So there is no inherent diversity which is the case with pure DS. We can see that hybrid DS/SFH has the highest throughput. Besides, we see that the maximum throughput for both SFH and DS is almost equal, however the throughput for SFH does not vary much over the large range of $G$. In the case of SFH with this parameter setting, the number of hopping frequencies is
$\mathrm{D}$ [slots]

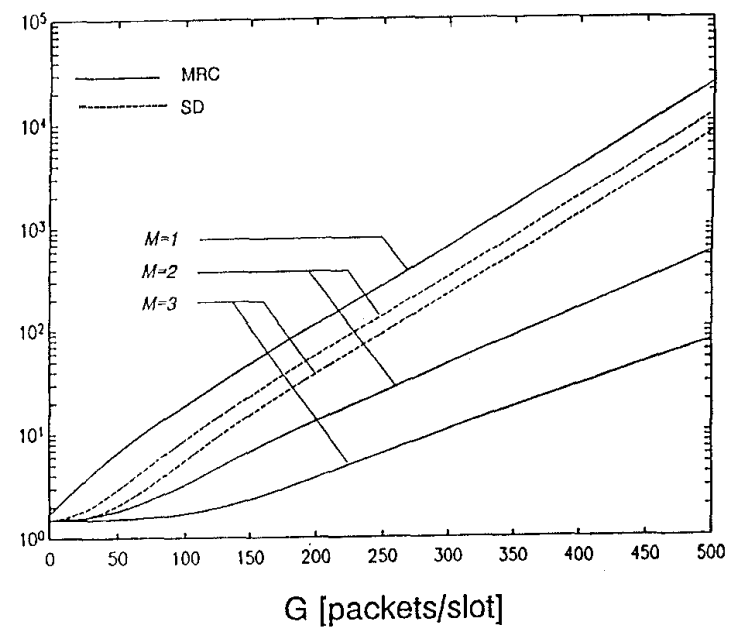

Fig. 4. Effect of diversity on the delay performance of hybrid DS/SFH CDMA with $B$-PSK with $M$ as a parameter, using the analytical channel model. $N_{b}=127 ; L=3$.

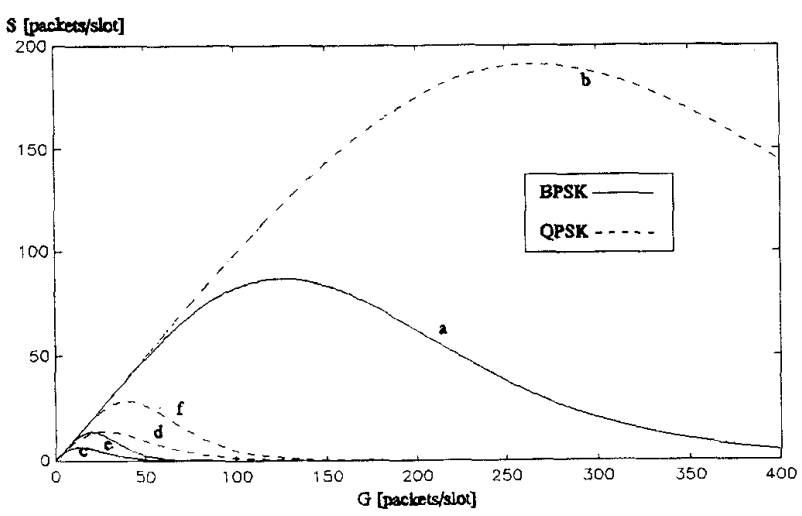

Fig. 5. Throughput of hybrid DS/SFH CDMA versus the DS CDMA without FEC coding, using the analytical channel model: (a) Hybrid: $L=3, M=4, q=10, N=127$; (b) Hybrid: $L=3, M=4, q=10, N=255$; (c) $L=21, M=1, q=1, N=1270$, DS; (d) $L=21, M=1, q=1, N=2550$, DS; (e) $L=21, M=4, q=1, N=1270$, DS; (f) $L=21, \bar{M}=4, q=1, \bar{N}=2550, \overline{\mathrm{DS}}$.

large enough to have a slightly better performance than DS, although the interference terms according to (16) and (17) are larger than in case of DS. The results obtained here are such that the possibilities of SFH will be investigated further in future work.

\section{B. Channel Model Using the Pico Cellular Measured Power Delay Profiles}

Results are presented in the following figures for the measured pico cellular channel model. In Figs. 7 and 8, respectively, the throughput and delay of hybrid DS/SFH CDMA has been compared with the throughput of DS CDMA for a fixed bandwidth $(80 \mathrm{MHz})$ in the $2.4 \mathrm{GHz}$ band using the measured pico cellular channel model. It is seen that hybrid DS/SFH CDMA is superior to DS CDMA. 


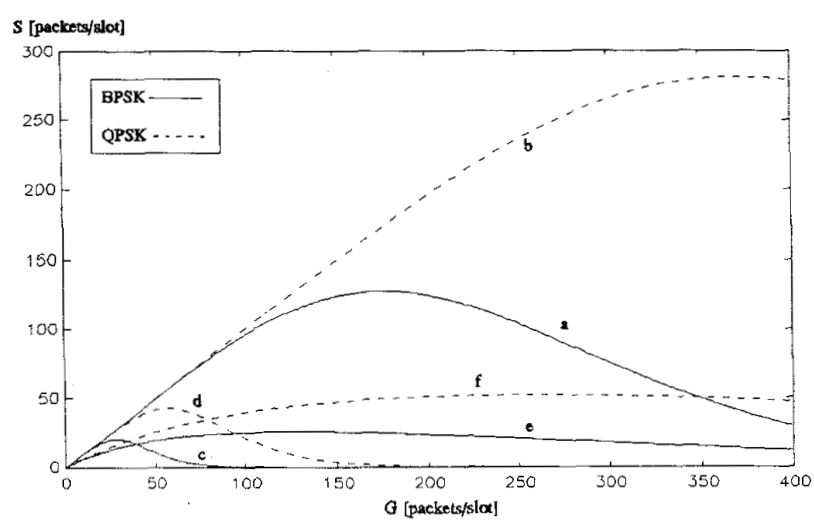

Fig. 6. Throughput of hybrid DS/SFH CDMA versus the DS CDMA and SFH CDMA with FEC coding, using the analytical channel model: (a) Golay coding, Hybrid: $L=3, M=4, q=10, N=63,(23,12)$; (b) Golay coding, Hybrid: $L=3, M=4, q=10, N=127$.(23.12); (c) Golay coding, DS: $L=21, M=4, q=1, N=630,(23,12)$; (d) Golay coding, DS: $L=21, M=4, q=1, N=1270,(23.12)$; (e) Golay coding. SFH: $L=1, M=4, q=630, N=1,(23,12)$; (f) Golay coding, SFH: $L=1, M=4, q=1270, N=1,(23,12)$.

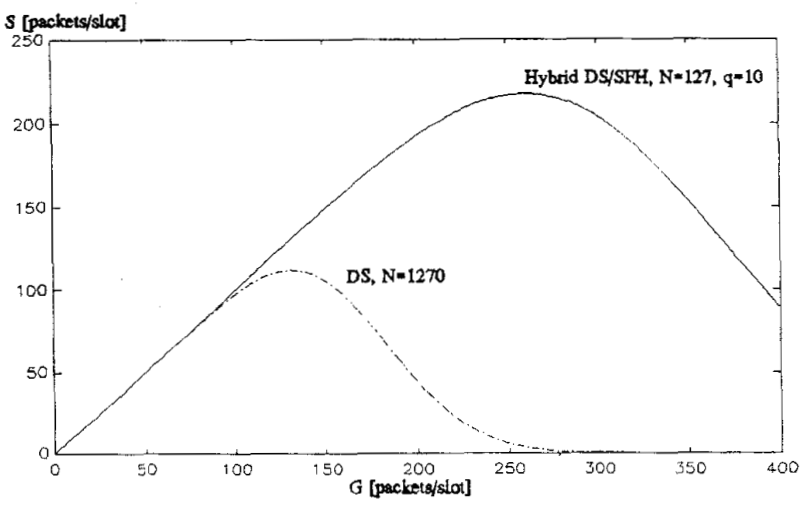

Fig. 7. Throughput comparison between hybrid DS/SFH CDMA and DS CDMA with $B$-PSK modulation, for a bandwidth of $80 \mathrm{MHz}$ in the 2.4 $\mathrm{GHz}$ band, using the measured pico cellular channel model. $K=400$, bandwidth $=80 \mathrm{MHz}, R_{b}=64 \mathrm{~Kb} / \mathrm{s}$, and SNR $=20 \mathrm{~dB}$.

Figures 9 and 10, respectively, depict the throughput and delay performance of a hybrid DS/SFH CDMA system for a fixed number of hopping frequencies $(q=6)$ and different values for the bit rate $R_{b}$ and $N$. We can see that the highest throughput is obtained with $R_{b}=125 \mathrm{~kb} / \mathrm{s}$ and $N=255$. A high bit rate combined with a smaller spreading sequence length yields a worse throughput and higher delays.

In Figs. 11 and 12, respectively, the throughput and delay of hybrid DS/SFH CDMA, using the measured pico cellular channel model has been compared with the performance obtained using the analytical model. We can see that the maximum throughput obtained using the MPD channel model is higher than in case of the analytical IID channel model. This is due to the fact that in the IID channel model it is assumed that all paths arrive with the same power at the receiver, as opposed to the MPD channel where delayed paths arrive with less power at the receiver than the first arriving path. This means that in case of the MPD channel the multipath interference is less than in case of the IID channel model,

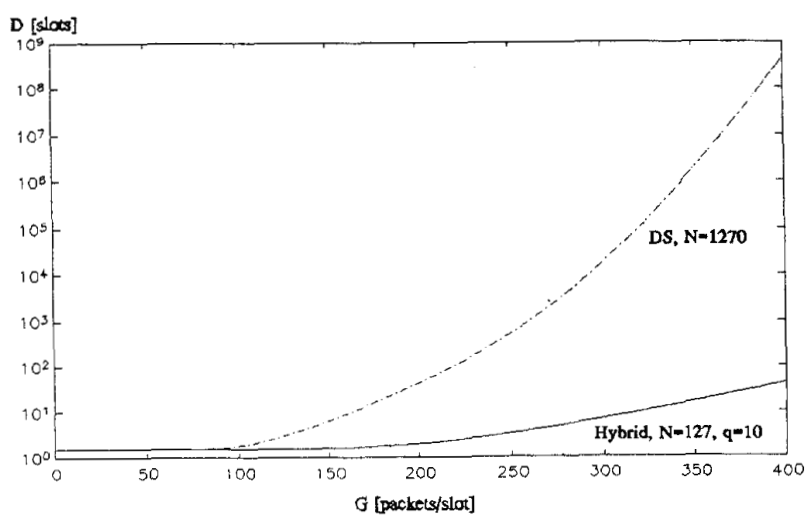

Fig. 8. Delay comparison between hybrid DS/SFH CDMA and DS CDMA with B-PSK modulation, for a bandwidth of $80 \mathrm{MHz}$ in the $2.4 \mathrm{GHz}$ band, using the measured pico cellular channel model. $K=400$, bandwidth $=80$ $\mathrm{MHz}, R_{b}=64 \mathrm{~Kb} / \mathrm{s}$, and $\mathrm{SNR}=20 \mathrm{~dB}$.

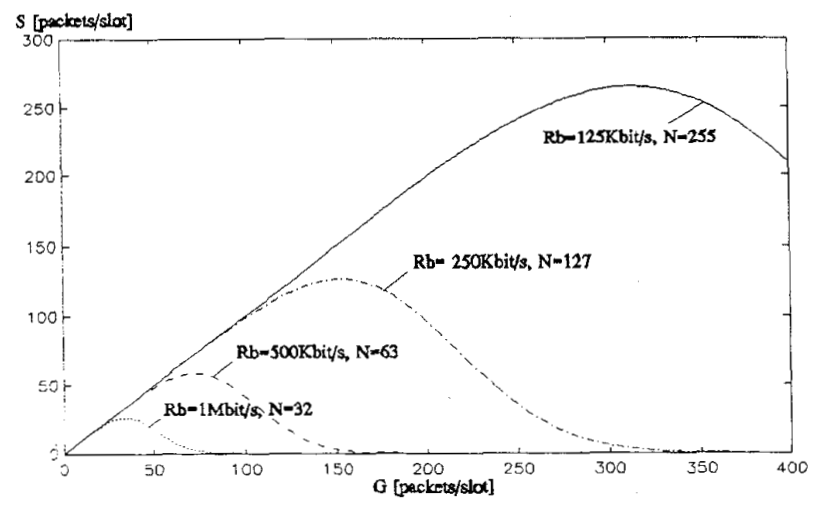

Fig. 9. Infuence of $R_{b}$ and $N$ on the throughput of hybrid DS/SFH CDMA with $B$-PSK modulation, at a fixed number of hopping frequencies $q$, using the measured pico cellular channel model. $K=400$, bandwidth $=200 \mathrm{MHz}$ $\mathrm{SNR}=20 \mathrm{~dB}$, and $q=6$

which yields a better performance than the case when using the IID model.

\section{CONCLUSION}

A performance analysis of hybrid DS/SFH, DS, SFH CDMA using analytical and measured pico cellular channels has been presented. The throughput and delay characteristics of hybrid DS/SFH CDMA has been compared with DS CDMA and SFH CDMA for analytical channels. The performance of hybrid DS/SFH CDMA has been compared for $Q$-PSK, $B$ PSK, and $D$-PSK modulation, using the analytical channel model. The performance of hybrid DS/SFH CDMA with $B$-PSK modulation has been compared for analytical and measured pico cellular channels.

The comparison of hybrid DS/SFH with $D-, B$-, and $Q$-PSK under the constraint of a fixed bandwidth has shown that $Q$ PSK yields the best and D-PSK the worst performance of the three modulation methods. With the demodulation of $D$-PSK modulated signals more noise is involved than demodulating $B$ - and $Q$-PSK modulated signals, which degrades the performance. In case of $Q$-PSK, the spreading code sequence 


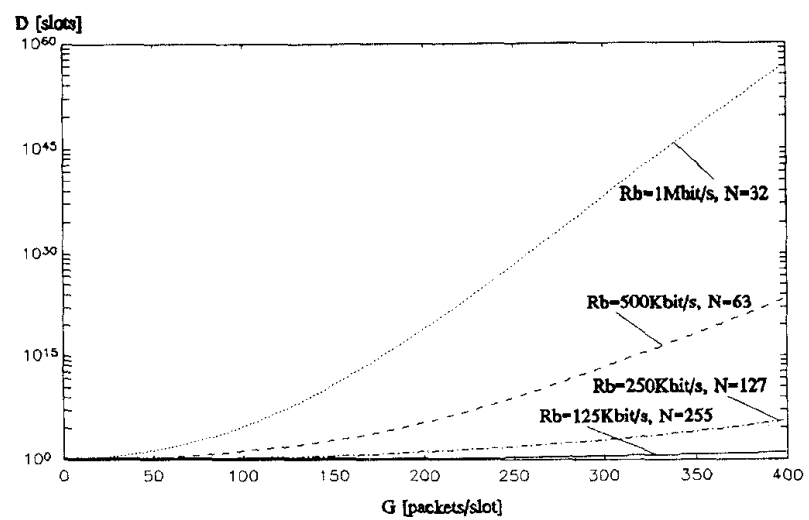

Fig. 10. Influence of $R_{b}$ and $N$ on the delay of hybrid DS/SFH CDMA with $B$-PSK modulation, at a fixed number of hopping frequencies $q$, using the measured pico cellular channel model. $K=400$, bandwidth $=200 \mathrm{MHz}$, $\mathrm{SNR}=20 \mathrm{~dB}$, and $q=6$.

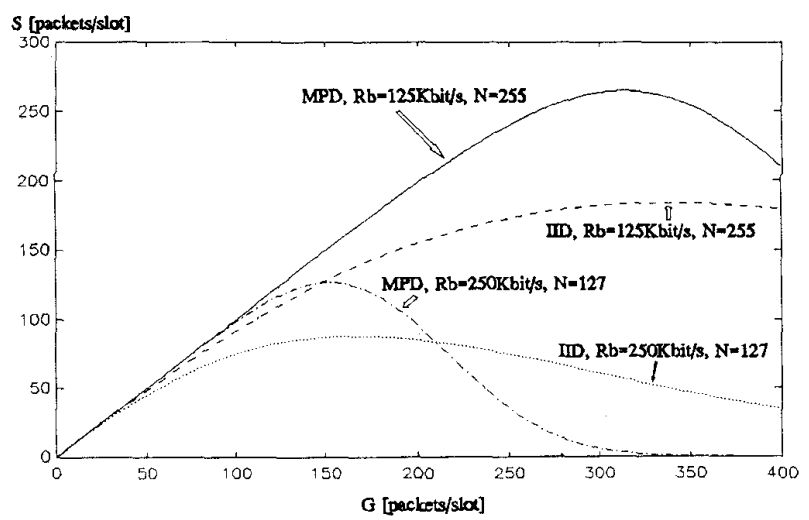

Fig. 11. Throughput comparison of hybrid DS/SFH CDMA with $B$-PSK modulation, using the measured pico cellular channel model (MPD) and the analytical channel model (IID) for two different bit rates. $K=400$, bandwidth $=200 \mathrm{MHz}$, SNR $=20 \mathrm{~dB}$, and $q=6$.

$N$ is twice as large as in case of B-PSK in order to fulfill the bandwidth constraint. This yield a better performance for systems using $Q$-PSK than systems using $B$-PSK.

With the constraint of a fixed bandwidth and bit rate a tradeoff can be made between the number of frequencies in the hopping pattern $q$ and the spreading code length $N$. Hybrid DS/SFH outperformes DS and SFH only with the combination of a relatively large number of hopping frequencies and a relatively small spreading code sequence. If we use relativly small bandwidths and high bitrates, then DS outperformes hybrid DS/SFH. This is due to the fact that at a small bandwidth the capabilities of hybrid DS/SFH, such as a relatively large number of hopping frequencies can not be fully explored. Additionally higher bitrates involve a higher number of resolvable paths, which increases the multipath interference. In this case, the relatively high length of the spreading code of DS in that case yield a better performance than the combination of a relatively low number of hopping frequencies and a relatively low spreading code length.

The use of FEC coding can enhance the performance in some cases. If the multi-user and multipath interference is

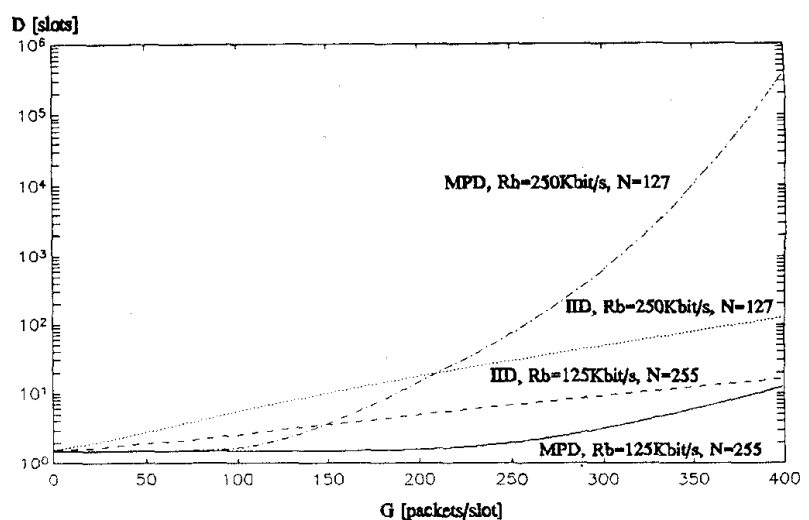

Fig. 12. Delay comparison of hybrid DS/SFH CDMA with $B$-PSK modulation, using the measured pico cellular channel model (MPD) and the analytical channel model (IID) for two different bit rates. $K=400$, bandwidth $=200$ $\mathrm{MHz}, \mathrm{SNR}=20 \mathrm{~dB}$, and $q=6$.

relatively large, which is the case for a large number of resolvable paths, the use of FEC coding only improves the performance if the code rate is large enough.

When using measured delay profiles the performance in terms of throughput and delay is better than the performance obtained with the analytical model. The difference performance using the two models is due to the difference in interference power. When using the MPD channel model the paths arrive with different power at the receiver, as opposed to the case of the analytical model. The analytical channel model in this case describes the worst case performance and yields a lower bound of the throughput and delay performance.

\section{REFERENCES}

[1] R. Prasad, H. S. Misser, and A. Kegel, "Performance analysis of directsequence spread-spectrum multiple-access communication in an indoor Rician fading channel with D-PSK modulation," Electron. Lett., vol. 26, pp. 1366-1367, Aug. 16, 1990.

[2] H. S. Misser, C. A. F. J. Wijffels, and R. Prasad, "Throughput analysis of CDMA with $D$-PSK modulation and diversity in indoor Rician fading radio channels," Electron. Lett., Vol. 27, no. 7, pp. 601-602, Mar. 28 1991.

[3] R. Prasad, C. A. F. J. Wijffels, and K. L. A. Sastry, "Performance analysis of slotted CDMA with $D$-PSK modulation, diversity and $\mathrm{BCH}$ coding in indoor radio channels," $A E U$, vol. 46, no. 6, pp. 375-382, 1992.

[4] C. A. F. J Wijffels, H. S. Misser, and R. Prasad, "A micro-cellular CDMA system over slow and fast Rician fading radio channels with forward error correction coding and diversity," IEEE Trans. Veh. Technol., vol. 42, pp. 570-580, Nov. 1993.

[5] R. Prasad, H. S. Misser, and A. Kegel, "Performance evaluation of direct-sequence spread-spectrum multiple-access for indoor wireless communications in a Rician fading channel," IEEE Trans. Commun., vol. 43, pp. 581--592, Feb./Mar./Apr. 1995.

[6] M. Kavehrad and B. Ramamurthi, "Direct-sequence spread spectrum with $D$-PSK modulation and diversity for indoor wireless communications," IEEE Trans. Commun, vol. COM-35, pp. 224-236, Feb. 1987.

[7] J. Wang and M. Moeneclay, "Hybrid DS/SFH spread-spectrum multipleaccess with predetection diversity and coding for indoor radio," IEEE J. Select. Areas Commun., vol. 10, no. 4, pp. 705-713, May 1992.

[8] __ "Hybrid DS/SFH SSMA with predetection diversity and coding over indoor radio multipath Rician-fading channels," IEEE Trans. Commun., vol. 40, no. 10, pp. 1654-1662, Oct. 1992.

[9] E. A. Geraniotis, "Noncoherent hybrid DS/SFH spread-spectrum multiple access communications," IEEE Trans. Commun., vol. COM-34, pp. 862-872, Sept. 1986. 
[10] A. Polydoros and J. Silvester, "Slotted random access spread-spectrum networks: An analytical framework," IEEE J. Select. Areas Commun., vol. SAC-5, no. 6, pp. 989-1002, July 1987.

[11] G. J. M. Janssen and R. Prasad, "Propagation measurements in an indoor radio environment at $2.4 \mathrm{GHz}, 4.75 \mathrm{GHz}$ and $11.5 \mathrm{GHz}$," in $42 n d$ Veh. Technol. Society VTS Conference Frontiers of Technology, Denver, Colorado, May 10-13, 1992, pp. 617-620.

[12] J. G. Proakis, Digital Communications, 2nd edn. New York: McGrawHill, 1989.

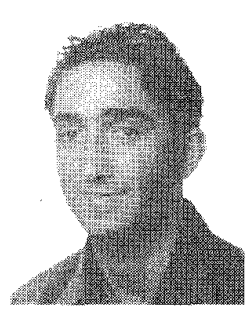

Fevzi Çakmak (S'96) was born on June 3, 1970, in Istanbul, Turkey. He received the M.Sc. degree from the faculty of electrical engineering of Delft University of Technology, The Netherlands, in 1995.

During his study, he was a trainee at the International Cooperation Department of Teléfonica de España. In 1995, he joined the Network and Service Control Department of KPN Research. His activities and interests there were in the areas of mobile communications, B-ISDN signaling using SVC's, B-ISDN standardization, ATM and TINA In 1996 he joined CMG Telecommunications and Utilities BV, where he is working on GSM.

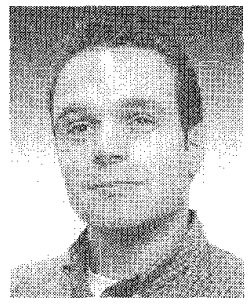

René G. A. Rooimans (M'93) was born in Rotterdam, The Netherlands, on May 3, 1967. He received the B.Sc.E.E. degree from Delft University of Technology, Delft, The Netherlands, in 1989 and 1993, respectively.

He worked as a Research Fellow at the Telecommunications and Traffic-Control Systems Group, Delft University of Technology, Delft. He is currently with the Telecommunications and Information Systems Department of EZH, one of the four Power Utility Companies in The Netherlands. His research interests are in the field of radio and mobile communications and spreadspectrum communications.

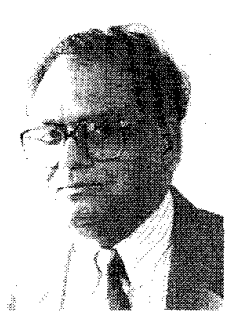

Ramjee Prasad (M'88-SM'90) was born in Babhnaur (Gaya), Bihar, India, on July 1, 1946. He received the B.Sc.(Eng) degree from Bihar Institute of Technology, Sindri, India, and the M.Sc.(End.) and Ph.D. degrees from Birla Institute of Technology (BIT), Ranchi, India, in 1968, 1970, and 1979 , respectively.

In 1970, he joined BIT as a Senior Research Fellow and became an Assistant Professor in 1980. While he was with BIT, he supervised many research projects in the area of microwave and plasma engineering. From 1983 to 1988, he was with the University of Dar es Salaam (UDSM), Tanzania, where he became a Professor in Telecommunications with the Department of Electrical Engineering, in 1986. At UDSM, he was responsible for the collaborative project "Satellite Communications for Rural Zones" with the Eindhoven University of Technology, The Netherlands. Since February 1988, he has been with the Telecommunications and Traffic-Control Systems Group, Delft University of Technology (DUT), The Netherlands, where he is actively involved in the area of personal indoor and mobile radio communications (PIMRC). His current research interests lies in wireless networks, packet communications, multiple access protocols, adaptive equalizers, spread-spectrum CDMA systems, and multimedia communications. $\mathrm{He}$ is currently involved in the European ACTS project FRAMES (Future Radio Wireband Multiple Access System) as a Project Leader of DUT, and is a member of a working group of European cooperation in the field of scientific and technical research (Cost-231) project dealing with "Evolution of Land Mobile Radio (including personal) Communications." He has served as a member of advisory and program committees of several IEEE international conferences. He has also presented keynote speeches, invited papers, and tutorials on PIMRC at various universities, technical institutions, and IEEE Conferences. He has published over 200 technical papers, and is the coordinating editor and one of the Editors-in-Chief of a Kluwer International Journal on Wireless Personal Communications; he is also a member of the editorial boards of other international journals including the IEEE Communications Magazine and the IEE Electronics and Communications Engineering Journal.

Dr. Prasad is listed in the US Who's Who in the World. He was Organizer and Interim Chairman of the IEEE Vehicular Technology/Communications Society Joint Chapter, Benelux Section. He is now the Elected Chairnan of the Joint Chapter. He is also founder of the IEEE Symposium on Communications and Vehicular Technology (SCVT) in the Benelux and he was the Symposium Chairman of SCVT'93 and SCVT'95. He is a Fellow of the IEE, a Fellow of the Institution of Electronics \& Telecommunications Engineers, a Member of the New York Academy of Sciences, and a Member of The Netherlands Electronics and Radio Society (NERG). 